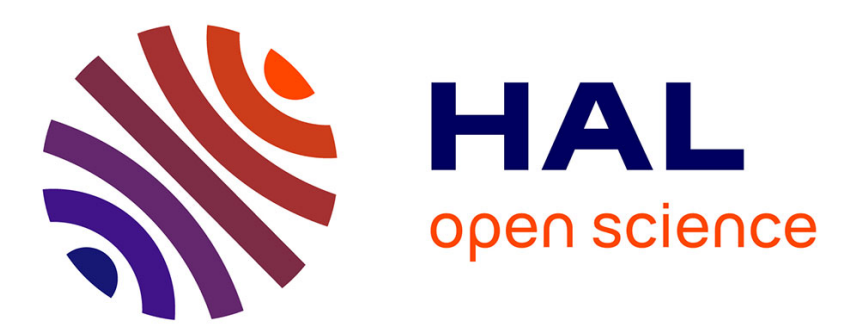

\title{
Navigating in a Virtual 3D Maze: Body and Gravity, Two Possible Reference Frames for Perceiving and Memorizing
}

Manuel Vidal, Alain Berthoz

\section{- To cite this version:}

Manuel Vidal, Alain Berthoz. Navigating in a Virtual 3D Maze: Body and Gravity, Two Possible Reference Frames for Perceiving and Memorizing. Spatial Cognition and Computation, 2005, 5, pp.139 - 161. 10.1080/13875868.2005.9683801 . hal-01519789

\section{HAL Id: hal-01519789 \\ https://hal-amu.archives-ouvertes.fr/hal-01519789}

Submitted on 9 May 2017

HAL is a multi-disciplinary open access archive for the deposit and dissemination of scientific research documents, whether they are published or not. The documents may come from teaching and research institutions in France or abroad, or from public or private research centers.
L'archive ouverte pluridisciplinaire HAL, est destinée au dépôt et à la diffusion de documents scientifiques de niveau recherche, publiés ou non, émanant des établissements d'enseignement et de recherche français ou étrangers, des laboratoires publics ou privés. 


\title{
Navigating in a Virtual 3D Maze: Body and Gravity, Two Possible Reference Frames for Perceiving and Memorizing
}

\author{
Manuel Vidal and Alain Berthoz \\ CNRS / Collège de France
}

Although recent studies have brought new insight into the mechanisms of spatial memory and cognitive strategies during navigation, most of these studies have concerned two-dimensional navigation and little is known regarding the problem of three-dimensional (3D) spatial memory. We found previously that memorizing complex 3D-structured corridors was easier with natural self-motion that included only yaw turns, and vertical translations facing the walls at vertical sections. This suggests that when only sideways (yaw) mental rotations had to be performed in order to shift from the experienced egocentric to the allocentric reference frame where recognition was tested, memorization of such corridors was improved. In the present investigation we studied the effect of tilting separately subject's body axis and self-motion's rotation axis relative to gravity. With a computerized 3D reconstruction task of the maze, we examined whether having any single rotation axis was enough to facilitate this reference shift or, if not, what aspect of the terrestrial condition-where visual displacement rotation, gravity and body axes were aligned-led to better performance. Field dependent (FD) and independent (FI) subjects, as determined by the rod and frame test, showed distinct effects of the navigation conditions. The FD group performance was markedly impaired when gravity and body axis were in conflict, independently of the rotation axis, whereas FI performance only slightly worsened when the body was tilted and the rotation axis remained aligned with gravity. Moreover, tilting the body in the control condition only worsened performance for the FD group.

Keywords: Spatial memory, reference frames, human, 3D maze, VR.

Correspondence concerning this article should be addressed to Manuel Vidal, Laboratoire de Physiologie de la Perception et de l'Action, Collège de France; email: manuel.vidal@college-de-france.fr. 


\section{Introduction}

\section{Human Navigation and Three-Dimensional Spatial Problems}

Human spatial navigation involves the updating of spatial information, accompanied by the development of spatial knowledge. Spatial updating is based on both the integration of one's self-motion and the recognition of environmental landmarks along the way, thus allowing one to retrieve one's relative position and then readjust for the errors predicted by the integration of kinaesthetic cues. The visual and other sensory information processed is received according to an egocentric frame of reference. Its successive memorization along a trajectory associated with landmarks is often called route knowledge. Once many distinct paths of a given environment are familiar, landmarks allow these routes to be connected by transformation to allocentric frames of references, and survey knowledge of the environment emerges.

Although recent investigations have brought new insight into the mechanisms of spatial memory and cognitive strategies during navigation, most of them concerned two-dimensional navigation. These studies were mostly restricted to planar spatial configurations while subjects stood upright with regard to the external reference provided by gravity. In such conditions only the azimuth corresponding to yaw turns has to be integrated to solve spatial tasks. Little is known regarding the problem of spatial 3D memory, despite the fact that it assumes great importance in modern societies. Going from one point to another inside a building is a typical situation requiring spatial 3D processing by the brain, and it occurs in everyday life. Navigation in weightlessness inside a space station is another example which, though far less frequent, is useful in trying to elucidate the underlying processes concerning both the use of a distinct selfmotion mode and the use of gravity as a reference frame. Only a few studies have addressed the issue of elevation during navigation and how the brain might process it. Gärling et al. (1990) studied the encoding and recall of the elevation of landmarks in a city by asking subjects to estimate from memory the difference in elevation between famous landmarks. The results showed that low precision information on elevation could be retrieved, and that it was not through a "mental travel process" between landmarks because decision times were not correlated with the distance separating them. It suggests that the estimation of elevation is independent of the horizontal dimensions. Montello and Pick (1993) used a pointing task to compare, either within or between layers, the learning of the spatial configuration of landmarks along two distinct paths of a university's superimposed floors. They found that the pointing performance was slower and less accurate between than within layers. In fact the mental representation of the landmarks' spatial configuration for each layer was correct, and subjects could establish links between layers, although it was harder than within one specific layer. These results support the notion that the human brain cannot easily construct 3D cognitive maps, and that navigating inside buildings probably generates specific cognitive maps for each $2 \mathrm{D}$ layer. This 
suggests a clear difference in the way information is processed and stored depending on whether it relates to the vertical or the horizontal dimension.

We shall now introduce some findings from animal electrophysiology experiments that are relevant to this topic. The neural activity associated with 3D navigation in weightlessness was recently studied (Knierim, McNaughton, \& Poe, 2000). A modified Escher staircase was used in orbital flight (corresponding to a complex 3D path ending at the exact position of the starting point). Recordings of rats' hippocampal place cells revealed that the animals' representational system remained unimpaired: after six $90^{\circ}$ turns, alternating leftward and upward, place cells associated with the start of the maze were firing again, as if they 'knew' they had come back to the starting point. These results have to be carefully considered since they are inconsistent with recent findings on head direction cells of rats (Stackman, Tullman, \& Taube, 2000), namely that they discharge according to a preferred direction of the head alignment's projection in a gravitationally horizontal plane and independently of its pitch orientation. In weightlessness the horizontal plane associated with head direction cells is probably reoriented onto the surface the animal is walking on.

\section{Considerations on Reference Frames}

Describing the multiple representations of space in the brain, Arbib (1991) introduced the problem by stating that "The representation of this quotidian space [of everyday action] in the brain is not one absolute space, but rather a patchwork of approximate spaces (partial representations) that link sensation to action." This points out two important features of the brain: firstly, that there are many different spaces adapted to specific sensory input and motor output, each one involving different reference frames and, secondly, that these representations are not precise. It is probably the redundancy resulting from the overlapping of the multiplicity of spaces, concerning a particular problem, that allows a fairly accurate estimation and processing of the problem.

There is a good deal of electrophysiological evidence from rats in support of the notion of multiple reference frames handled by the brain. On the one hand, there are the place cells of the hippocampus that discharge when the animal is near a certain place. It has been shown that the place associated with these cells can be defined in terms of a specific location but also in terms of a goal, landmark or starting position that can move relatively to the external reference frame (Gothard, Skaggs, Moore, \& McNaughton, 1996). With practice, place cells were also able to learn, on a rotating platform, to distinguish places from two reference frames: a rotating one relevant to the foraging task, and a static one relevant to the stable surroundings (Bures, Fenton, Kaminsky, \& Zinyuk, 1997; Zinyuk, Kubik, Kaminsky, Fenton, \& Bures, 2000). On the other hand, there are the head direction cells that discharge when the head is pointing in a specific direction. They can also be defined according to distinct reference frames mainly guided by vision (Zugaro, Tabuchi, \& Wiener, 2000)—inside a cylindrical arena, head direction cells are defined in terms of the cylinder wall reference frame, but when removed from this environment they are defined with 
reference to the room (Zugaro, Berthoz, \& Wiener, 2001). Studies on contraversive pushing in spatial neglect patients suggest that subjective body orientation is disturbed because of the cortical structures responsible for transforming sensory inputs into a cohesive reference frame for interpretation (Karnath, 1994), although gravity inputs seem not to interact with the orientation judgment, the bias being defined according to an egocentric reference frame (Karnath, Fetter, \& Niemeier, 1998). These studies support the hypothesis that many distinct reference frames can be handled by the brain for specific processing, with sensory information being transformed for each specific use. This is often the case in motor control, where the brain can call upon many different reference frames according to the motor task. For instance, in a pointing task in 3D space it has been shown that a viewer- rather than an elbowcentered reference frame is used (McIntyre, Stratta, \& Lacquaniti, 1997).

In a previous investigation (Vidal, Amorim, \& Berthoz, 2004), we studied the effect of the relationship between egocentric and allocentric frames of references on memorization of complex 3D-structured environments in which the subjects were passively driven. The environment's spatial structure represented for instance buildings with several floors or a space station. Different conditions were compared, inspired by navigation in terrestrial, subaquatic and weightless elements. In the terrestrial navigation condition, self-motion included yaw rotations and vertical translations facing the walls at vertical sections as in an elevator, whereas in the weightless navigation condition subjects could move along or turn about any axis. The task was to recognize among four successive outside views of corridors the one that had been traveled. In order to perform this task, participants had to create a mental image or representation of the environment structure while moving inside it. Since perception was done in an egocentric reference frame and the recognition task in an allocentric reference frame, a reference shift had to be performed while exploring, so as to build the mental image segment by segment. The results showed that in the terrestrial condition, where only one mental rotation (in this case the yaw) had to be performed to shift from an egocentric to an allocentric reference frame, memorization of the corridors was improved both in accuracy and in reaction time. This is consistent with an investigation on map reading for piloting, which found that the simpler the relationship between the map reference frame and the environment to be explored, the easier the spatial orientation (Péruch \& Lapin, 1993).

\section{From Mental Rotations to Cognitive Maps}

In order to understand the problematic of the investigations presented here, it is of interest to introduce findings on mental rotations. First of all, mental rotation of patterns involves rotation of a reference frame rather than rotation of a template-like representation (Robertson, Palmer, \& Gomez, 1987). Easton \& Sholl (1995) found that relationship between objects of a 2D-array from imagined viewpoints is retrieved by means of a body-centered coordinate system, thus requiring imagined continuous body translation or rotation. This is 
consistent with the literature on mental rotation of displays: many studies have reported that performance in spatial updating of an object array was significantly better after imagined viewer rotation than after imagined object rotation (for a review, see Wraga, Creem, \& Proffitt, 1999, 2000). Wraga et al. explain this discrepancy in terms of the difficulty in the imagined array rotation that stems from inherent problems in performing cohesive rotations of all components of the intrinsic representation. In contrast, when the viewer moves, the relative reference frame is automatically and naturally updated. Another explanation could be that mental transformation of images requires, at least partially, motor processes in the brain: a motor dual-task by means of a joystick improved the performance of the mental rotation of the image when the two rotations were compatible (Wexler, Kosslyn, \& Berthoz, 1998), and the object's imagined rotation nearly reached the viewer level of performance when rotations included haptic information (Wraga, Creem, \& Proffitt, 2000).

Returning to our task described above, adding properly each segment to the mental representation while exploring the corridor also required the extraction of spatial relations after translation and rotations (which direction does the next turn take?). Therefore, the mental construction was also done by imagining one's rotation inside the currently built representation. The mental rotation involved in the egocentric to allocentric shift was easier in the terrestrial condition because rotations were only about one axis, corresponding to yaw rotations. But the rotation axis was aligned with two other axes defining two reference frames: the observer's main body axis and the gravity axis. In the current investigation we tried to ascertain the contribution of each of these alignments to the capacity to perform the mental rotation involved in the process of memorizing the corridor's structure. In the first experiment (called the ground experiment) we tilted these axes separating the alignment influences, and in the second experiment (called the space experiment) we simply suppressed the influence of the gravity reference frame.

\section{Aims of the Present Investigation}

The findings of a previous investigation revealed that, in a natural terrestrial self-motion condition that required mental rotations around only one axis (yaw) to update the representation of the environments, performance was better than when rotations around the three canonical axes were required. In the present study, we tried to answer two questions. The first question was whether simply having to process a single rotation axis is sufficient to make the mental representation updating easier, or if it has to be a particular axis. Since the single rotation axis of the terrestrial condition was aligned with both the main body and gravity axes, the second question was which reference frame contributes the most to improving the cognitive processes involved in memorizing a 3D maze.

On the one hand, we know that once body and gravity references are brought into conflict by having the subjects lie down on their sides, some subjects' performance will be affected by this conflict. For that reason, each subject's field dependency was previously determined with the classic rod and frame test, 
and we expected to find correlations between this factor and a subject's performance in the main task when lying down. On the other hand, we wondered whether aligning the self-motion's unique rotation axis with the body or with gravity would lead to better results. In the first case, rotations around the body axis (yaw turns) are, from an ecological point of view, the most natural and frequent situations, and therefore, although gravity was in conflict, they could be properly interpreted. In turn, the second situation actually occurs in real life: imagine watching somebody walking on TV while you are lying down on a couch. Even though this situation is less frequent, the consistency of self-motion with respect to gravity could be sufficient to allow the brain to interpret such situations without ambiguity.

Considering mental rotations, Shiffrar and Shepard (1991) showed that performance was improved when the axes of the object, rotation, and gravitational vertical were aligned. Tilting one of them resulted in a deterioration of both the speed and accuracy of the mental rotation. Based on these results, we formulated the following hypothesis for the first question above: if the subject remains upright, tilting the rotation axis will impair the mental updating process. Imagining rotations in the transverse plane (yaw rotation) independently of the body orientation was always better for viewer rather than array imagined rotations (Creem, Wraga, \& Proffitt, 2001). The viewer advantage was lost only when the rotation was in the coronal plane (roll rotation). In another study, a clear independence of body vs. gravity orientation was also found for imagining roll rotation of objects disposed in a cubic array (Oman et al., 2002). Therefore, when it is possible to imagine rotations of the environment around the observer's body axis, manipulations of the egocentric reference frame are more efficiently performed. This suggested for our experiment the following hypothesis for the second question above: conditions where rotations are consistent with the body reference frame lead to the best performance independently of the gravity reference frame. This hypothesis implies that the rotation axis aligned with the body axis will provide better results than if it is aligned with gravity.

\section{Method}

\section{Participants}

Sixteen naïve subjects (six women and ten men) aged from 19 to 34 years participated in this investigation. Most of them were studying at university in various fields and levels. All except two were right-handed. They all gave written consent before starting and were paid for taking part in the experiment. 


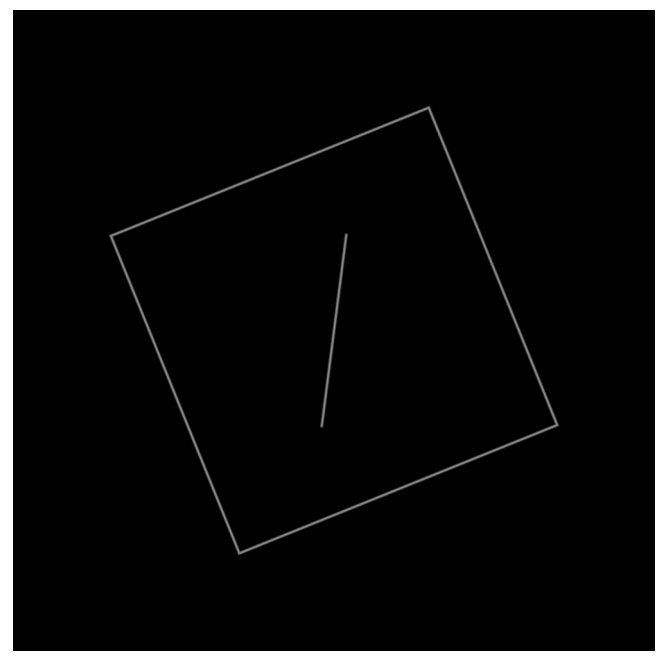

Figure 1. A view of the rod and frame test as experienced by subjects. The rod was randomly tilted from the vertical, and the frame was tilted by either $22^{\circ},-11^{\circ},+11^{\circ}$ or $+22^{\circ}$. Subjects had to adjust the rod with the keyboard's left and right arrows until they felt it was perfectly vertical, the adjustment precision being $0.1^{\circ}$.

\section{Computerized Rod and Frame Test}

In order to look for a correlation between performance in our spatial task and the well-known individual differences concerning the influence of a visual frame on the subjective vertical (Asch \& Witkin, 1948), subjects previously underwent a computerized rod and frame test. They were shown a tilted rod centered inside a tilted frame (see Figure 1).

The rod was randomly tilted from the vertical leftward or rightward at an angle ranging from $4^{\circ}$ to $8^{\circ}$, the frame was tilted by either $-22^{\circ},-11^{\circ},+11^{\circ}$ or $+22^{\circ}$. Subjects had to adjust the rod with the keyboard's left and right arrows until they felt it was perfectly vertical. A single key touch increased or decreased the rod's tilt-angle by $0.1^{\circ}$, and a continuous pressure increased or decreased the rod's tilt-angle by $3 \%$. Two blocks of 12 trials corresponding to three adjustments for each frame's tilt-angle were performed, with a pause between the two blocks. Before each trial, a fixation point appeared during $500 \mathrm{~ms}$ in the center of the screen followed by a dark screen in order to guide the subjects' direction of gaze. We ensured that the border of the screen could not be used as a visual reference by taking two precautions: firstly, the only source of light was 
the rod and the frame, and their luminosity was set to a low level; secondly, the frame was in the peripheral vision, and subjects were asked to keep their gaze in the center of the screen. The rod and frame test lasted about 5 minutes.

\section{Experimental Set-Up}

Subjects faced a large screen either seated on a chair of adjustable height or lying on their sides on a specially constructed bed in a $90^{\circ}$-roll position. In both situations, the line of sight was centered on the large screen, on which the simulated virtual self-motions were projected (apparatus detailed in Figure 2). The answers were given with a keyboard and the sounds played through headphones worn by the subjects. In order to avoid any influence of the subjects' body position on keyboard handling, when they were seated it was placed on their knees, and when they were lying down it was vertically fixed at the same distance and orientation with respect to their arms.

\section{Procedure}

Each trial of the experiment included a visual navigation phase followed by a reconstruction task. During the navigation phase, subjects were passively driven at a constant speed through a virtual cylindrical 3D corridor made of stones. A static view showed an avatar at the beginning of the corridor for $1000 \mathrm{~ms}$ before visual motion started (see Figure 3). The segments constituting the corridors were the same length and were aligned with one of the canonical axes (see Figure 4). Six different navigation conditions (detailed in the Experimental Conditions section) were compared in 10 different corridors, half being

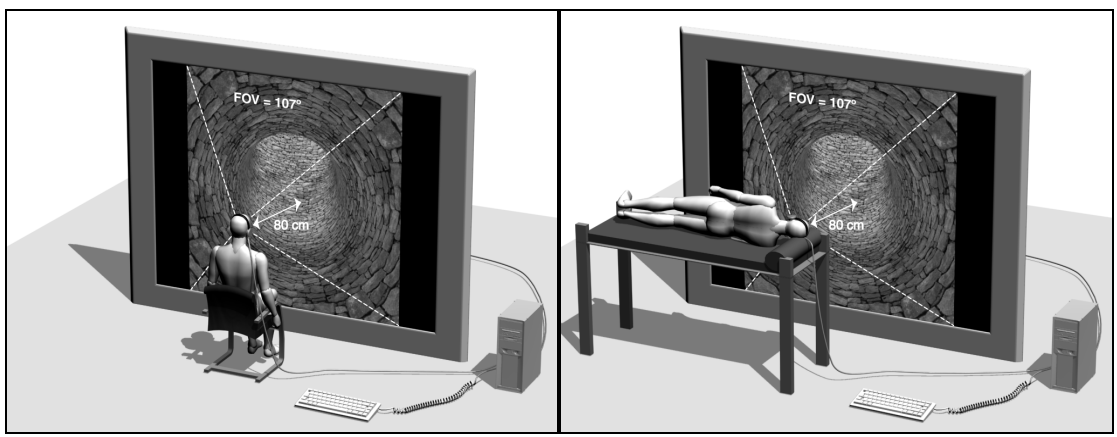

Figure 2. The experimental set-up for the seated upright conditions (left) and the lying down conditions (right). Subjects' line of sight was centered on a translucent screen with a $107^{\circ}$ horizontal and vertical field of view. They interacted using a keyboard and wore headphones. A PC computer equipped with a GeForce 2 video card rendered the simulated virtual self-motions retroprojected onto the screen, and played the dual-task sounds. The squared resolution was 1200x1200 pixels at a frame rate of $85 \mathrm{~Hz}$. 


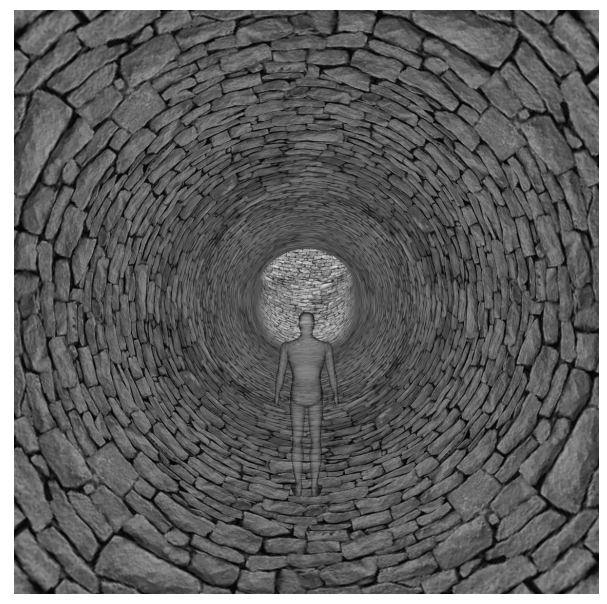

Figure 3. The static inside view with the avatar displayed at the beginning of the exploration of the corridor. The avatar has the same body orientation as the subjects and gives an indication for the reconstruction referential. The perspective correction was adjusted to the real field of view experienced by the subjects.

randomly selected from a 4-segment corridor database and the other half from a 5-segment database (see Appendix for details concerning the databases' construction).

During the reconstruction task, subjects were asked to draw with the computer the remembered 3D shape of the corridor. They were first shown an external view of the first segment with an avatar at the entrance point indicating the orientation relative to which the reconstruction had to be made. This avatar, like the one shown at the beginning of the navigation phase, represented the observer. It was aligned with the subjects' body position. Therefore, when they were in the upright position the avatar was vertical, and when they were in the lying down position the avatar was horizontal (see Figure 4). Four red arrows, labelled from 1 to 4 , indicated the four possible directions of the next segment. Each segment was reconstructed by pressing the key corresponding to the label of the red arrow chosen. Once the correct number of segments had been entered, a message appeared asking the subject to validate the drawing by pressing the spacebar key. At any time, subjects could cancel their last choice by pressing the backspace key.

The full experiment for a given subject consisted of two sessions of 30 trials each, divided into blocks of 10 trials. One of the sessions was performed sitting upright and included the three corresponding navigation conditions (see paragraph below), the other was performed lying down in a $90^{\circ}$-roll position and included the other three navigation conditions. The order of the sessions was 

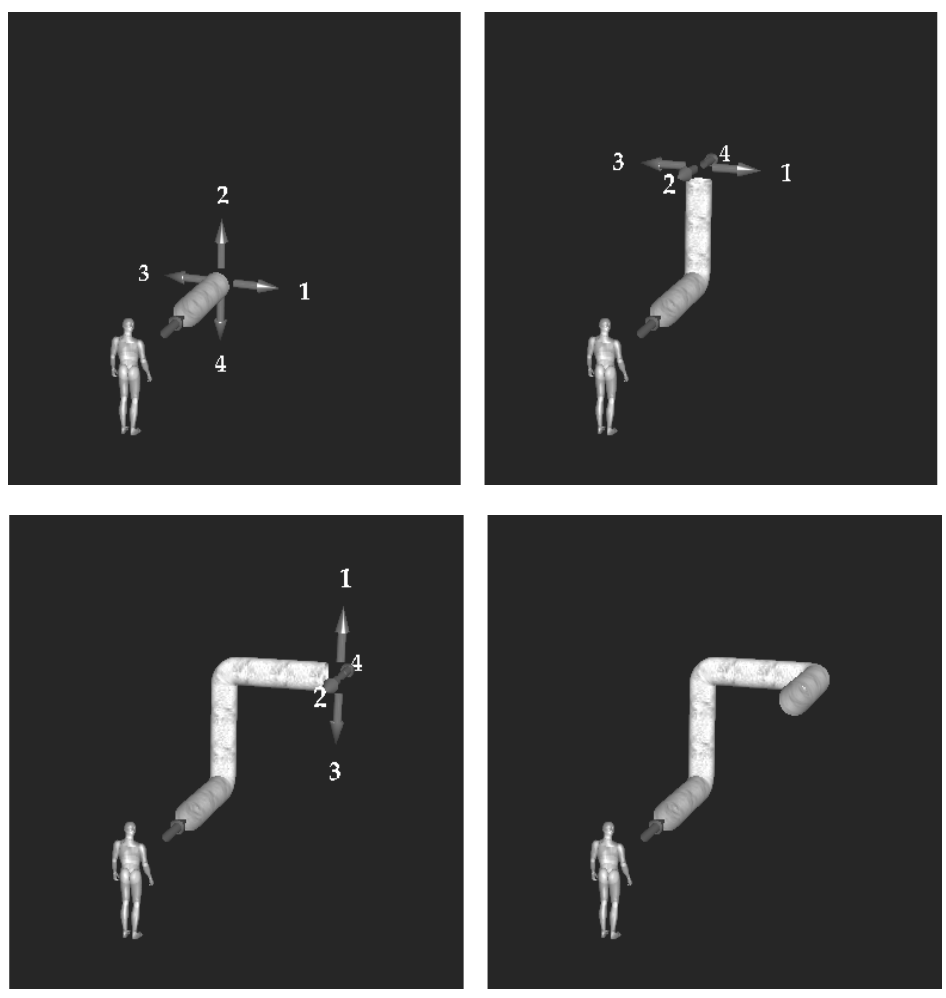

Figure 4. The outside view during the reconstruction task. The subjects had to choose segment-by-segment between the four possible directions for the next segment, each direction being parallel to one of the canonical axes X, Y and Z. Once the correct number of segments had been entered, a message appeared asking the subject to validate the drawing by pressing the spacebar key. Subjects could cancel their last choice at any moment by pressing the backspace key.

counterbalanced between subjects. Each session started with six practice trials, for each of the three navigation conditions defined in the corresponding two body position. Subjects could then learn how to use the computer interface. The task being cognitively very demanding, the two sessions for any given subject were performed on different days in order to avoid saturation. After each block of 10 trials, a score showing the average accuracy at reproduction was displayed followed by a 5-minute pause. This feedback was given in order to keep subjects motivated during the whole experiment. Subjects triggered each trial by pressing a specific key when ready. The full experiment lasted approximately two hours. 


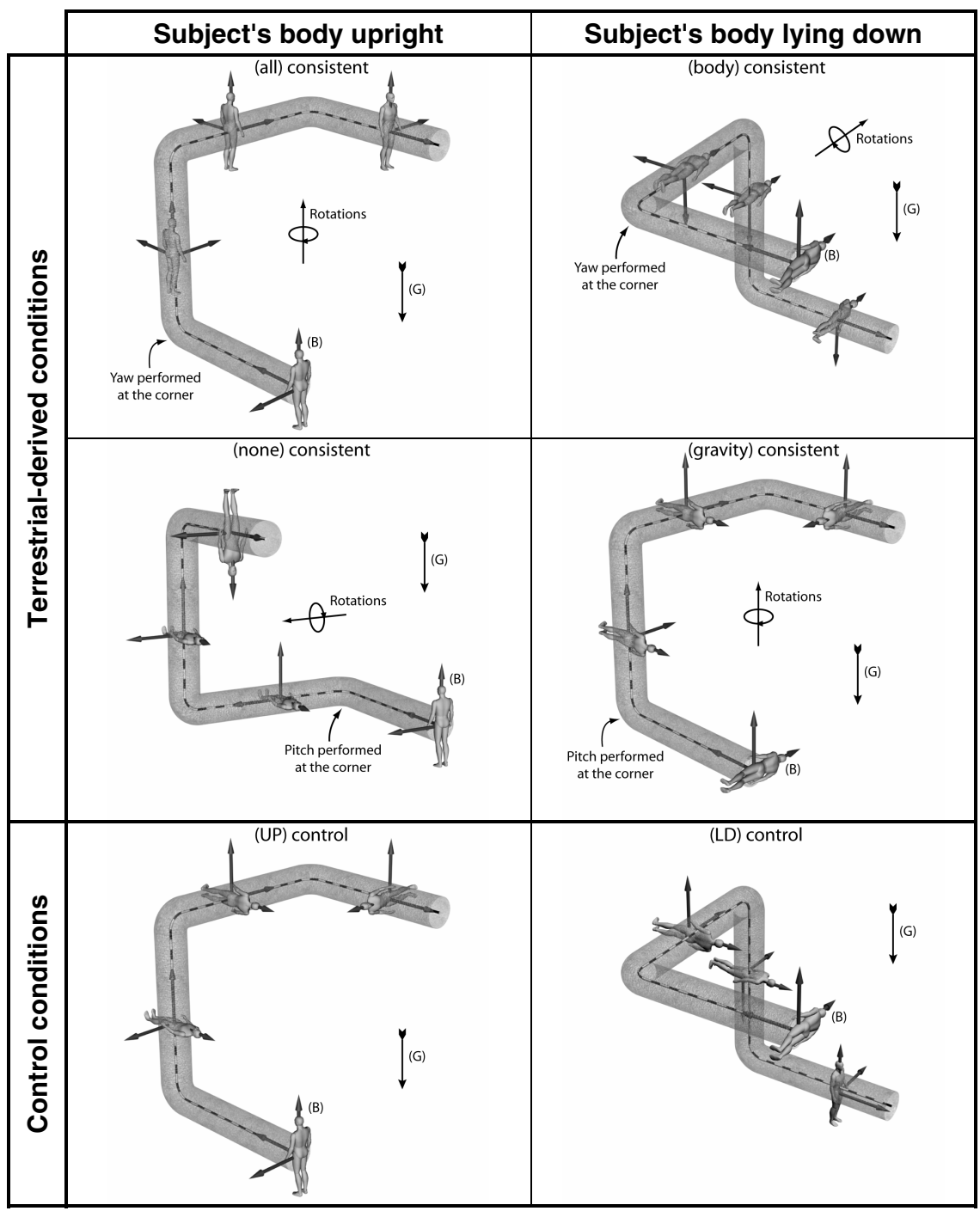

Figure 5. These illustrations show the virtual body orientation in each segment of the same corridor, according to the six studied navigation conditions. Four derived from natural terrestrial navigation: all, none, body and gravity conditions named according to the consistency of self-motion's rotations with the body (B) and gravity $(\mathrm{G})$ reference frames. In the upright UP and lying down LD control navigation conditions, simulated self-motion included both yaw and pitch turns. The body reference frame is characterized by the initial virtual position in the corridors, the latter matching subject's body position. 


\section{Verbal Dual-Task}

According to the model of working memory proposed by Baddeley in 1986 and validated since (Baddeley, 1998b), short-term memory is composed of two "slave" systems for storing and maintaining visuospatial and verbal information, piloted by the central executive system that processes the stored information, allocating attentional and cognitive resources. The first system, called the visuospatial sketchpad (VSSP), used for mental imagery manipulations (Pearson, Logie, \& Green, 1996; Bruyer \& Scailquin, 1998), is also involved in high-level comprehension and reasoning tasks that involve spatial representations such as motion simulation (Salway \& Logie, 1995) and mental simulations of mechanisms (Sims \& Hegarty, 1997). All these investigations showed the VSSP to be largely independent of the verbal system consisting of the phonological and articulatory loop, and recent studies using functional imagery techniques have shown that mental imagery tasks and verbal tasks are processed in different regions of the brain (Baddeley, 1998a). In order to avoid processed in different regions of the brain (Baddeley, 1998a). In order to avoid memorization of a verbal sequence of the directions taken in corridors, subjects were required to perform a dual-task consisting of a verbal working memory load. Our task involves high-level manipulations of spatial representations and is therefore processed by the VSSP, which is largely independent of the verbal working memory. Loading the verbal memory would therefore prevent its use as an alternate encoding strategy for memorising the shape of the corridor. At the very beginning of each trial, three random numbers between 20 and 59 were played through the headphones and subjects had to memorise them in the correct order. Just after the reconstruction task, subjects had to recall this sequence of numbers, and an immediate sound feedback was played if more than one number was incorrect or not in the correct order.

Although the storage capacity of verbal working memory is usually greater than three items, we thought this would be sufficient to prompt the spatial storage strategy. An audio presentation of the numbers was used rather than a visual presentation in order to avoid visual memorization in the VSSP.

\section{Experimental Conditions}

Six navigation conditions were studied, four derived from a natural terrestrial condition where a single rotation axis is used in the simulated self-motion and two control conditions where rotations about the three canonical axes are used (see Figure 5). In all conditions, two different reference frames were engaged: the gravity reference frame and the body's reference frame. The four terrestrialderived conditions characterized by their unique axis of rotation were defined according to the consistency of this particular axis with the body and gravity reference frames. The two control conditions were used as performance references, and are defined only according to the alignment of the body axis and gravity. 
The six navigation conditions were created in this way:

all Navigation condition where self-motion's rotation axis is aligned with both the body axis and gravity (subjects are seated upright). This condition corresponds to the natural terrestrial navigation condition.

none Navigation condition where self-motion's rotation axis (90.0 tilted) is aligned neither with body axis nor with gravity, but the body axis is aligned with gravity (subjects are seated upright).

body Navigation condition where self-motion's rotation axis is horizontal and aligned with the body axis (subjects are lying down in a $90^{\circ}$-roll position).

gravity Navigation condition where self-motion's rotation axis is vertical and thus aligned with gravity, but the body axis is horizontal (subjects are lying down in a $90^{\circ}$-roll position).

UP Control navigation condition in which subjects are seated upright.

LD Control navigation condition in which subjects are lying down.

In the all and body consistent conditions, the head was always kept upright and in vertical segments the walls scrolled up or down in front of the subject as if inside a transparent elevator. Before entering a vertical segment a yawrotation was done (indicated in the Figure 5) in order to orient the sight to the direction taken after going up or down; in this way subjects knew which direction was coming next. In the UP and LD control conditions, the viewing direction pointed towards the end of the current segment and at each junction a single yaw- or pitch-rotation was performed to reorient the line of sight with the next segment, therefore allowing the three rotations of the 3D space. In all conditions gaze-orientation rotated in anticipation of each turn as would occur in natural conditions (Grasso, Glasauer, Takei, \& Berthoz, 1996; Wann \& Swapp, 2000; Wann et al., 2000). Linear speed was kept constant during the whole selfmotion simulation.

\section{Data Analysis}

For each trial, the total reconstruction latency and definition of the corridor and the answers to the dual-task were recorded. For each trial, a reconstruction score for the drawn corridor was calculated, corresponding to the number of segments reconstructed correctly from the beginning, excluding the first segment, divided by the total number of segments of the corridor minus one. For instance, if the corridor had 5 segments and the first three segments only were correct the reconstruction score would be $(3-1) /(5-1)=50 \%$. The chance level of this score for a random reconstruction is $10.9 \%$ and $8.3 \%$ for a 4 - and 5-segment corridor, respectively, giving an average chance level of $9.6 \%$ for balanced groups of trials containing the same number of 4- and 5-segment corridors. A score for the dual-task (DT score) was also calculated, and corresponded to the number of 
correct numbers in the correct order divided by 3 . For instance, if the given sequence was 23-57-31, the answers 23-56-31 and 57-23-31 would each obtain a score of $66.6 \%$.

A 2 (field dependency group) $\times 2$ (number of segments) $\times 6$ (navigation condition) ANOVA design table was used. The field dependency group (field dependent (FD) and field independent (FI)) being considered as a betweensubjects factor, while number of segments (4 and 5) and navigation condition (all, none, body, gravity, UP and LD) were the within-subjects experimental factors. The dependent variables were the reconstruction score and latency, and the dual-task score. Post-hoc analyses were performed with the Scheffé test when possible, and with a planned comparison when there was an interaction with the field dependency group between-subjects factor.

\section{Results}

\section{Rod and Frame Results}

The average deviation from vertical reproduced for the four frame orientations was calculated for each subject $\left(\varepsilon_{ \pm 11^{\circ}}\right.$ and $\varepsilon_{ \pm 22^{\circ}}$ ). The $11^{\circ}$ tilted frame and $22^{\circ}$ tilted frame effects were calculated for each subject, corresponding to the deviation from the middle of leftward and rightward errors for each frame tilt angle:

$$
E_{11^{\circ}}=\frac{\left|\varepsilon_{+11^{\circ}}-\varepsilon_{-11^{\circ}}\right|}{2} \quad E_{22^{\circ}}=\frac{\left|\varepsilon_{+22^{\circ}}-\varepsilon_{-22^{\circ}}\right|}{2} \quad E_{\text {global }}=\frac{E_{11^{\circ}}+E_{22^{\circ}}}{2}
$$

The median values of the $11^{\circ}$ - and $22^{\circ}$-effect obtained were respectively $2.17^{\circ}$ and $2.04^{\circ}$. They were used to discriminate subjects: 8 subjects presented a $22^{\circ}$ and $11^{\circ}$-effect below these criteria and constituted the field-independent group (FI group, $\mathrm{n}=8, E_{\text {global }}=1.06^{\circ} \pm 0.55^{\circ}$ ); and 8 subjects presented a $22^{\circ}$ - and $11^{\circ}$ effect above these criteria and constituted the field-dependent group (FD group, $\mathrm{n}=8, E_{\text {global }}=3.65^{\circ} \pm 0.83^{\circ}$ ). We managed to have well-balanced groups with regard to the body position of the starting session: each group had 4 subjects that started seated upright and 4 subjects that started lying down.

\section{Qualitative Results}

Subjects reported that the task was very demanding and that they had to maintain a high level of concentration in order to perform it properly. Despite the difficulty of the task, the level of performance was rather good. As Figure 6 shows, accuracy at the reconstruction of the corridor was far above the chance level. Some of the subjects who appeared to be field dependent according to the rod and frame test said they experienced great difficulty in performing the task in the lying down conditions. They were highly confused about what reference to use for both memorizing and reconstructing: they knew the reconstruction was with reference to their body but experienced some conflicting interference with the gravity reference frame. These subjective remarks were correlated with the performance presented in the Results section and will be discussed later. 
Table 1

Reconstruction Score (Mean \pm SE) for Different Navigation Conditions and Grouped by Field Dependency Factor

\begin{tabular}{|c|c|c|c|c|c|c|c|}
\hline \multirow[t]{2}{*}{ Group } & \multirow[t]{2}{*}{$n$} & \multicolumn{3}{|c|}{ Body Upright } & \multicolumn{3}{|c|}{ Body Lying Down } \\
\hline & & all & none & UP & body & gravity & LD \\
\hline $\begin{array}{l}\text { Field } \\
\text { Independent }\end{array}$ & 8 & $\begin{array}{l}77.60 \pm \\
5.59\end{array}$ & $\begin{array}{l}67.92 \pm \\
6.23\end{array}$ & $\begin{array}{l}70.84 \pm \\
6.58\end{array}$ & $\begin{array}{l}83.03 \pm \\
4.41\end{array}$ & $\begin{array}{l}69.17 \pm \\
5.70\end{array}$ & $\begin{array}{l}76.88 \pm \\
5.89\end{array}$ \\
\hline $\begin{array}{l}\text { Field } \\
\text { Dependent }\end{array}$ & 8 & $\begin{array}{l}73.13 \pm \\
5.51\end{array}$ & $\begin{array}{l}53.76 \pm \\
3.66\end{array}$ & $\begin{array}{l}62.71 \pm \\
4.04\end{array}$ & $\begin{array}{l}44.80 \pm \\
10.81\end{array}$ & $\begin{array}{l}42.29 \pm \\
5.87\end{array}$ & $\begin{array}{l}34.27 \pm \\
5.49\end{array}$ \\
\hline All together & 16 & $\begin{array}{l}75.36 \pm \\
3.83\end{array}$ & $\begin{array}{l}60.84 \pm \\
3.94\end{array}$ & $\begin{array}{l}66.77 \pm \\
3.87\end{array}$ & $\begin{array}{l}63.91 \pm \\
7.49\end{array}$ & $\begin{array}{l}55.73 \pm \\
5.26\end{array}$ & $\begin{array}{l}55.57 \pm \\
6.74\end{array}$ \\
\hline
\end{tabular}

\section{Reconstruction Score}

The reconstruction performance (mean reconstruction score \pm standard error) grouped by field independent subjects, field dependent subjects and altogether for different navigation conditions are given in Table 1. The effect of the field dependency factor on each condition will be analyzed, and the performance of the different conditions will be compared. The control conditions are presented first, since they will be used as a reference for the lying down effect on both the field-dependent and field-independent group. Then, the four terrestrial-derived conditions (all, none, body, and gravity) will be analyzed.

\section{Comparison of Control Conditions}

The interaction between FD group and navigation condition for the UP and LD control conditions (see Figure 6) was significant, $F(1,14)=11.11, p<0.005$. In the upright position the FD and FI groups had the same average performance, with $62.7 \%$ and $70.8 \%$, respectively (no statistically significant difference), whereas in the lying down position the FD group had an average performance of $34.2 \%$ and the FI group had an average performance of $76.8 \%, F(1,14)=28.01$, $P<0.001$. The performance degradation of the FD group when lying down as compared to seated upright was highly significant, $F(1,14)=15.1 ; p<0.002$. The number of segments of corridors had a significant effect on both the UP condition, $F(1,14)=22.69, p<0.001$, and the LD condition, $F(1,14)=8.91$, $p<0.01$.

\section{Comparison of Terrestrial-Derived Conditions}

The average reconstruction scores of the FI and FD groups for the four terrestrial-derived conditions are plotted in Figure 6. As with the control conditions, only the conditions in the lying down position showed a significant effect of the field dependency factor: $83.0 \%$ for the FI group against $44.8 \%$ for the FD group for the body condition, $F(1,14)=10.72, p<0.006$, and $69.2 \%$ for the FI group against $42.3 \%$ for the FD group for the gravity condition 


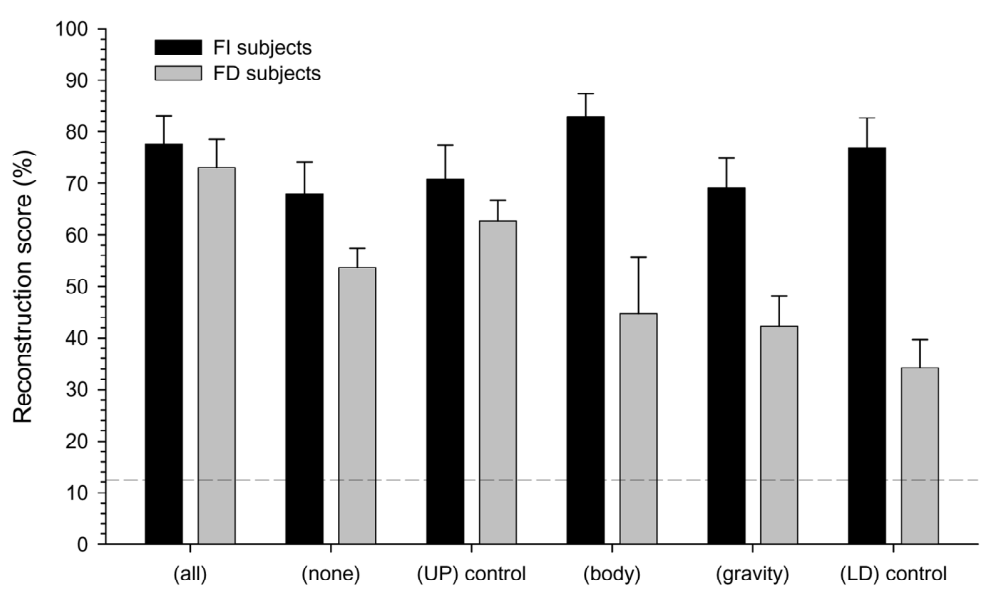

Upright conditions

Reclined conditions

Figure 6. Average reconstruction score (mean $+S E)$ for both field-dependent $(n=8)$ and field-independent $(n=8)$ groups, as a function of the navigation conditions. Dashed line represents the chance level.

$F(1,14)=10.80, p<0.006$. The FD group's performance was highly impaired when subjects were lying down, which was not the case for FI group. There was also a clear tendency separating the performance of the FI and FD groups for the none condition, $F(1,14)=3.84, p<0.07$.

The global performance for the all condition (with an average of 77.6\%) was higher than for the none condition (with an average of $60.8 \%, F(1,14)=9.54$, $p<0.008$ ), or for the body condition (with an average of $63.9 \%, F(1,14)=5.11$, $p<0.04$ ), and the gravity condition (with an average of $55.7 \%, F(1,14)=12.36$, $p<0.004)$. A planned comparison revealed that these differences resulted only from the FD group performance, the FI group showing no significant difference between the all condition and the others. There was no other significant difference between conditions, either globally or by group. The only tendency that should be highlighted, $F(1,14=3.45, p<0.09$, was the poorer performance of the FI group in the gravity condition (with 69.1\%) than in the body condition (with $83.0 \%$ ). In the FD group there was no difference between these conditions.

\section{Terrestrial-Derived Compared to Control Conditions}

Performance in the UP control condition was significantly below that of the all condition $F(1,14)=6.48, p<0.03$, with $66.8 \%$ and $75.36 \%$, respectively. This observation is consistent with the results of a previous study that compared subject's performance in these two navigation conditions (Vidal et al., 2004). A planned comparison revealed again that this difference resulted only from the FD group's performance, the FI group showing no statistical difference. This 


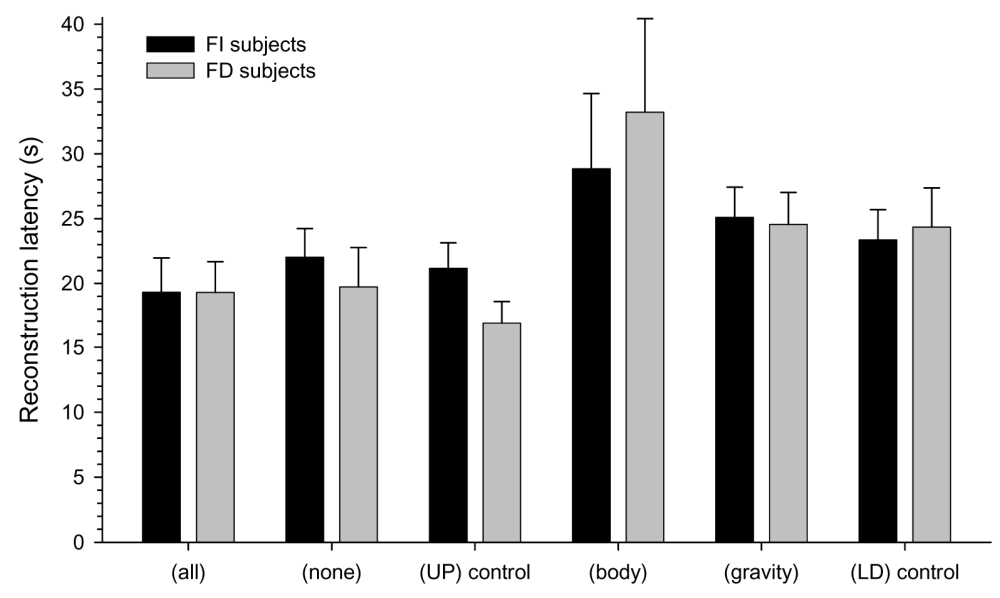

Upright conditions

Reclined conditions

Figure 7. Reconstruction latency (mean $+S E$ ) for both field-dependent $(n=8)$ and field-independent $(n=8)$ groups, as a function of the navigation conditions.

may well have been due to a smaller performance difference in this group that would require a greater number of subjects to become significant $(n>8)$. Apart from this, there was no difference between the terrestrial-derived conditions and the corresponding control conditions, either for the FD group or for the FI group. Therefore, removing the coherence of one of the reference frames for the terrestrial condition was enough to worsen the performance as compared to a navigation condition with no particular axis for the rotations.

\section{Reconstruction Latency}

Reconstruction latencies of subjects grouped by field dependency factor for different navigation conditions are given in Figure 7. Latencies were statistically shorter for upright conditions $(19.7 \pm 1.0 \mathrm{~s}$ for the all, none and UP conditions grouped) than for lying down conditions $(26.6 \pm 3.9 \mathrm{~s}$ for the body, gravity and LD conditions grouped), $F(1,14)=14.64, p<0.002$. A planned comparison showed that this difference was significant for the FD group, $F(1,14)=11.97$, $p<0.004$, but not for the FI group. Post-hoc analysis revealed that the only significant mean differences between conditions were as follows: the average latency for the body condition was longer than the average latency for the all $(p<0.002)$, none $(p<0.012)$, and UP $(p<0.002)$ conditions. 


\section{Dual-Task Performance}

The dual-task performance was fairly high with a mean score of $73.9 \pm 3.3 \%$, indicating that the subjects had effectively memorized and recalled the numbers. This means that the subjects' verbal working memory was at least partially loaded, and that their strategy for the main task could not entirely rely on the verbal memorization of the directions taken in the corridor. Interestingly, there was no statistical difference in the dual-task results, either across field dependency groups or across conditions, and for that reason they could not be used in the analysis as an indicator of the difficulty of the main task. In fact, this observation confirms the independence of the VSSP and the phonological loop, as mentioned above: although conditions had a noticeable effect on the spatial task, they did not necessarily make the verbal memorization of the numbers any harder. The differences in the accuracy of reconstruction observed in the different conditions must therefore have stemmed from the spatial processing of the navigation information.

\section{Discussion}

In the control conditions, we observed that, in the memorizing and recalling process for 3D corridors, lying down had a marked effect on FD subjects whereas there was no such effect on FI subjects. The horizontal control condition corresponds to a condition where body and gravity frames of reference are tilted, as compared to natural conditions where they share the same vertical axis. FD subjects were affected by this tilt and though there was no particular axis for this navigation condition, their performance was highly impaired. In contrast, FI subjects remained unaffected. This difference was also observed in the terrestrial-derived conditions: the field dependency factor had a significant effect only in conditions where subjects were lying down, and again with a marked deterioration in the performance of the FD group. As we expected, the introduction of an unnatural orientation of gravity by having the task performed lying down disturbed some subjects. Interestingly, the field dependency factor was correlated with this and allowed us to predict whether lying on his/her side would have an effect on a given subject's performance. For this reason, we will mostly be discussing the results of the FI and FD groups separately.

The accuracy at the reconstruction task indicates that, regardless of gravity orientation, FI subjects had the same performance level in the terrestrial-derived conditions where self-motion's rotations were consistent with their body reference frame, whereas it was slightly lower in conditions where it was tilted. Furthermore, for FI subjects, no difference was observed for vertical and horizontal control conditions, which also supports the notion that for this category of subjects the spatial memorization process is highly independent of gravity orientation with regard to body orientation. Although this difference did not reach statistical significance, only the processing time for reconstruction tended to be slightly higher when FI subjects were lying down, resulting from the handling of two conflicting reference frames, namely gravity and body. This 
is consistent with the well-known characteristics of field-independent subjects. In the rod and frame test they can adjust the rod to the vertical fairly precisely even though the visual field reference frame conflicts with both the gravity and body reference frames. Field-independent subjects can therefore select the most appropriate reference frame to use for a specific task and ignore any other conflicting reference frame, which explains why they are more capable of handling two conflicting reference frames.

In contrast, the accuracy of FD subjects at the reconstruction task was significantly lower in the two terrestrial-derived conditions where gravity is tilted with regard to the body reference frame (lying down position) as compared to the natural condition all, independently of the orientation of self-motion's rotation axis. When this unique rotation axis was tilted but standing upright, performance was also significantly lower than for the natural condition, but seemed slightly better than for the lying down terrestrial-derived conditions. A similar result was observed for the vertical and horizontal control conditions. As regards the reconstruction processing time for FD subjects, latencies were significantly longer for all lying down conditions. Therefore, for FD subjects, the spatial memorization process for a $3 \mathrm{D}$ maze is highly impaired when the body and gravity reference frames are not consistent. Moreover, the misalignment of the unique rotation axis in the upright condition also leads to a poorer performance. Again, this is consistent with the characteristics of fielddependent subjects. Despite the fact that in the rod and frame test they know the frame is tilted, their adjustments are not precise because they are influenced by the visual reference frame. They cannot ignore the conflicting reference frame in order to rely solely on the appropriate one, provided in this case either by gravity or by the body posture.

Another interesting finding was provided by the none condition, in which self-motion's unique rotation axis was $90^{\circ}$-roll tilted and subjects were seated upright. Although the body and gravity reference frames were consistent, the effect of field dependency on the measured performance accuracy almost reached significance, the FI group having better overall scores than the FD group. On the one hand, FI subjects had almost the same performance (no significant differences) as when the rotation axis was aligned with the other two axes (corresponding to the all condition). On the other hand, FD subjects had a significantly poorer performance than that observed in the all condition. It would appear that, even if the body and gravity reference frames are consistent, FD subjects are less capable of imagining rotations about an axis that is distinct from the body axis, whereas FI subjects can do this quite well.

If we consider all the subjects together, the answer to the first question, as to whether simply having to process a single rotation axis is sufficient to make updating the mental representation easier, is no, it has to be a particular axis. This is consistent with previously reported findings on mental rotations (Shiffrar $\&$ Shepard, 1991). This answer was clear for the FD group, but not so clear for the FI group, in which the recorded performance was poorer when the rotation axis was tilted, but not significantly so. Regarding the question of whether self- 
motion's rotations consistent with the body reference frame or with the gravity reference frame once the body is tilted with regard to gravity would lead to better performance, we found some unexpected results. Here again, FD subjects reacted differently from FI subjects. The FI group of subjects tended to prefer the alignment of the rotation axis with the body axis in order to perform the spatial task in such a condition, which is consistent with the fact that mental rotations are always better when there is the possibility of imagining rotation around the observer's body axis (Creem et al., 2001). In contrast, the FD group did not show any difference in accuracy between these conditions, both being equally impaired as compared to upright conditions. We would suggest that for FD subjects the problem of dealing with two conflicting reference frames took precedence over the expected preference for rotations around the body axis. Moreover, processing times for the condition where rotations were aligned with the body were longer, in particular for FD subjects, signifying that they needed more time to achieve the same level of performance in this condition as when rotations were around the gravity axis. A possible explanation for these surprising findings could be that in our experiment we displayed an avatar reminding subjects of the position of their body. This was done in order to orient the reconstruction task, where the avatar was also displayed at the entrance of the corridor. If subjects had taken this as a support for imagining rotations, it would have given preferential treatment to the condition where subjects were lying down and the rotations were around their body axis. In spite of this, the processing latencies of FD subjects were very long without any improvement in accuracy. If, instead, we had shown an avatar aligned with self-motion's rotation axis, FD subjects would probably have performed better in the condition where gravity was aligned with the rotation axis than in the condition where the body was aligned with the rotation axis. We consider that it would have been of interest had Creem and colleagues, in their study on physically impossible mental self-rotations (Creem et al., 2001), distinguished between FD and FI subjects. They concluded that transformations of egocentric reference frames are better when they consist of rotations around the observer's body axis. Our findings suggest that these conclusions might differ according to the field dependency groups.

\section{Conclusion}

In conclusion, reference frames involved in navigation have very distinct effects on the capacity to build a mental representation of the environment structure according to the field dependency factor. We know that the updating of such representations requires the capacity of the observer to imagine his/her rotations within the environment. Firstly, we found that the performance of FD subjects was markedly impaired when body and gravity were misaligned whereas that of FI subjects was not. Secondly, when the body was tilted with regard to gravity, FI subjects seemed to have a preference for self-motion where rotations were performed around the body axis, and this was not the case for field dependent 
subjects. Thirdly, even when body and gravity reference frames were consistent, tilting the rotation axis adversely affected the performance of FD subjects, but not that of FI subjects. To summarize, FD subjects cannot handle any kind of conflicting reference frame, the worst being a tilted body with regard to gravity. In contrast, FI subjects have a rather high level of performance for any situation involving conflicting reference frames. Field dependency, as determined by the classic rod and frame test, is a good indicator of performance whenever subjects are exposed to inconsistent frames of reference, field-independent subjects showing greater resistance to conflicts. Globally, the fact that self-motion's rotations were consistent with the body reference frame was probably the most important factor in the natural terrestrial navigation condition.

\section{Appendix - Construction of the Corridors}

The 4- and 5-segment corridor databases were built as follows. We firstly included all the possibilities in the databases $\left(4^{3}=64\right.$ and $4^{4}=256$, respectively). Then we removed all the cyclical corridors, and all those that had more than 2 successive turns in the same plane (either horizontal or vertical). In the end, the 4- and 5-segment databases had 28 and 88 different corridors, respectively.

\section{Acknowledgments}

I would like to thank particularly Stéphanie Cochin for supporting and helping me at all stages of this experimental work. I also would like to thank Michel Denis and Emilie Deyzac for their helpful advice on the design of the dual-task, Larry Young for his comments on the results, Nick Barton for proofreading the manuscript, and last but not the least all the students and friends who participated in the experiment.

\section{References}

Arbib, M. A. (1991). Interaction of multiple representations of space in brain. In J. Paillard (Ed.), Brain and space. Oxford: Oxford University Press.

Asch, S. E. \& Witkin, H. A. (1948). Studies in space orientation. II. Perception of the upright with displaced visual fields and with body tilted. Journal of Experimental Psychology, 38, 455-477.

Baddeley, A. (1998a). Recent developments in working memory. Current Opinion in Neurobiology, 8, 234-238.

Baddeley, A. (1998b). Working memory. Comptes Rendus de L'Academie Des Sciences. Serie III, Sciences de la Vie, 321, 167-173.

Bruyer, R. \& Scailquin, J. C. (1998). The visuospatial sketchpad for mental images: Testing the multicomponent model of working memory. Acta Psychologica, 98, 17-36.

Bures, J., Fenton, A. A., Kaminsky, Y., \& Zinyuk, L. (1997). Place cells and place navigation. Proceedings of the National Academy of Sciences of the United States of America, 94, 343-350. 
Creem, S. H., Wraga, M., \& Proffitt, D. R. (2001). Imagining physically impossible self-rotations: geometry is more important than gravity. Cognition, 81, 41-64.

Easton, R. D. \& Sholl, M. J. (1995). Object-array structure, frames of reference, and retrieval of spatial knowledge. Journal of Experimental Psychology: Learning, Memory, and Cognition, 21, 483-500.

Gärling, T., Böök, A., Lindberg, E., \& Arce, C. (1990). Is elevation encoded in cognitive maps? Journal of Environmental Psychology, 10, 341-351.

Gothard, K. M., Skaggs, W. E., Moore, K. M., \& McNaughton, B. L. (1996). Binding of hippocampal CA1 neural activity to multiple reference frames in a landmark-based navigation task. Journal of Neuroscience, 16, 823-835.

Grasso, R., Glasauer, S., Takei, Y., \& Berthoz, A. (1996). The predictive brain: Anticipatory control of head direction for the steering of locomotion. Neuroreport, 7, 1170-1174.

Karnath, H. O. (1994). Subjective body orientation in neglect and the interactive contribution of neck muscle proprioception and vestibular stimulation. Brain, 117, 1001-1012.

Karnath, H. O., Fetter, M., \& Niemeier, M. (1998). Disentangling gravitational, environmental, and egocentric reference frames in spatial neglect. Journal of Cognitive Neuroscience, 10, 680-690.

Knierim, J. J., McNaughton, B. L., \& Poe, G. R. (2000). Three-dimensional spatial selectivity of hippocampal neurons during space flight. Nature Neuroscience, 3, 209-210.

McIntyre, J., Stratta, F., \& Lacquaniti, F. (1997). Viewer-centered frame of reference for pointing to memorized targets in three-dimensional space. Journal of Neurophysiology, 78, 1601-1618.

Montello, D. R. \& Pick, H. L. J. (1993). Integrating knowledge of vertically aligned large-scale spaces. Environment and Behaviour, 25, 457-483.

Oman, C. M., Shebilske, W. L., Richards, J. T., Tubré, T. C., Beall, A. C., \& Natapoff, A. (2002). Three dimensional spatial memory and learning in real and virtual environments. Spatial Cognition and Computation, 2, 355-372.

Pearson, D. G., Logie, R. H., \& Green, C. (1996). Mental manipulation, visual working memory, and executive processes. Psychologische Beiträge, 38, 324-342.

Péruch, P. \& Lapin, E. A. (1993). Route knowledge in different spatial frames of reference. Acta Psychologica, 84, 253-269.

Robertson, L. C., Palmer, S. E., \& Gomez, L. M. (1987). Reference frames in mental rotation. Journal of Experimental Psychology: Learning, Memory, and Cognition, 13, 368-379.

Salway, A. F. \& Logie, R. H. (1995). Visuospatial working memory, movement control and executive demands. British Journal of Psychology, 86 ( Pt 2), 253-269.

Shiffrar, M. M. \& Shepard, R. N. (1991). Comparison of cube rotations around axes inclined relative to the environment or to the cube. Journal of Experimental Psychology: Human Perception and Performance, 17, 44-54. 
Sims, V. K. \& Hegarty, M. (1997). Mental animation in the visuospatial sketchpad: Evidence from dual-task studies. Memory \& Cognition, 25, 321332.

Stackman, R. W., Tullman, M. L., \& Taube, J. S. (2000). Maintenance of rat head direction cell firing during locomotion in the vertical plane. Journal of Neurophysiology, 83, 393-405.

Vidal, M., Amorim, M. A., \& Berthoz, A. (2004). Navigating in a virtual threedimensional maze: How do egocentric and allocentric reference frames interact? Cognitive Brain Research, 19, 244-258.

Wann, J. P. \& Swapp, D. K. (2000). Why you should look where you are going. Nature Neuroscience, 3, 647-648.

Wexler, M., Kosslyn, S. M., \& Berthoz, A. (1998). Motor processes in mental rotation. Cognition, 68, 77-94.

Wraga, M., Creem, S. H., \& Proffitt, D. R. (1999). The influence of spatial reference frames on imagined object- and viewer rotations. Acta Psychologica, 102, 247-264.

Wraga, M., Creem, S. H., \& Proffitt, D. R. (2000). Updating displays after imagined object and viewer rotations. Journal of Experimental Psychology. Learning, Memory, and Cognition, 26, 151-168.

Zinyuk, L., Kubik, S., Kaminsky, Y., Fenton, A. A., \& Bures, J. (2000). Understanding hippocampal activity by using purposeful behavior: Place navigation induces place cell discharge in both task-relevant and taskirrelevant spatial reference frames. Proceedings of the National Academy of Sciences of the United States of America, 97, 3771-3776.

Zugaro, M. B., Berthoz, A., \& Wiener, S. I. (2001). Background, but not foreground, spatial cues are taken as references for head direction responses by rat anterodorsal thalamus neurons. Journal of Neuroscience, 21, RC154.

Zugaro, M. B., Tabuchi, E., \& Wiener, S. I. (2000). Influence of conflicting visual, inertial and substratal cues on head direction cell activity. Experimental Brain Research, 133, 198-208. 